\title{
Cardiac surgery-associated acute kidney injury: tools for enriching clinical trial populations
}

\author{
Andrew D. Shaw, MB, FRCA, FFICM, FCCM
}

Received: 18 April 2017 / Accepted: 8 May 2017/Published online: 12 May 2017

(c) Canadian Anesthesiologists' Society 2017

The term acute kidney injury (AKI) has become synonymous with an acute decline in renal function, even though the kidneys themselves are almost never "acutely injured". When AKI occurs soon after heart surgery, it is called cardiac surgery-associated AKI (CS-AKI), and because of its frequency (up to $50 \%$ depending on the definition used and the population studied), ${ }^{1,2}$ it has become an outcome of great interest to clinicians and researchers alike. Many efforts have been made to achieve consensus in definition - most recently converging on the "Kidney Disease: Improving Global Outcomes (KDIGO)" definition $^{3}$ recently reviewed by Schetz- ${ }^{4}$ and controversy exists about whether urine output criteria always increase the diagnostic precision for this definition system. ${ }^{5}$

Despite great attention to CS-AKI, almost no progress has been made in its prevention or treatment, and this is a source of ongoing frustration. In an era when research funding is increasingly difficult to obtain, we need to find ways to increase the chances that the studies we do fund are successful. One way to achieve this is to include only patients with proven disease in prospective intervention trials. In this way, we avoid those who do not have disease and thus who cannot possibly benefit. Furthermore, costs are reduced and we increase the chance of finding an effective treatment. Nevertheless, this also removes the possibility of prevention, which is generally a more desirable "intervention", especially if we are able to identify a group of patients at increased risk of developing the disease in question. Perhaps the ideal scenario is one

\footnotetext{
A. D. Shaw, MB, FRCA, FFICM, FCCM ( $₫)$

Department of Anesthesiology, Vanderbilt University Medical Center, 1211, 21st Avenue South, Medical Arts Building, Room 722, Nashville, TN, USA

e-mail: andrew.shaw@vanderbilt.edu
}

where we can prospectively identify a patient population at high risk of developing an outcome and then include only these patients in the clinical trial. Zarbock et al. ${ }^{6}$ have shown that this approach can be successful in CS-AKI through use of a combination of clinical enrichment and urinary biomarker measurements.

In this issue of the Journal, Karkouti et al. ${ }^{7}$ report an observational study that uses an immediate postoperative rise in serum creatinine to predict a subset of patients that later develops CS-AKI. The design of the study is robust, and most importantly, the investigators used an entirely independent dataset to validate their work. This removes much of the usual concern about model overfitting, external generalizability, and relevance in the "real world", especially since the validation dataset is derived from an earlier reported multicentre pragmatic clinical trial. ${ }^{8}$ In the present study, the authors report that a $30 \%$ immediate (i.e., at the time of intensive care unit [ICU] admission) increase in serum creatinine identifies a population of patients at high risk of developing AKI in the next $48 \mathrm{hr}$. The positive predictive value in the validation set was $55 \%$ (i.e., there is about a one in two chance that someone with a $30 \%$ increase in serum creatinine on ICU admission will develop AKI in the next $48 \mathrm{hr}$ ), and the negative predictive value was $92 \%$ (i.e., it is almost a certainty that someone without a $30 \%$ rise will not develop AKI). This knowledge could be very useful for investigators planning intervention trials in CS-AKI. If we can essentially guarantee an event rate of $50 \%$ in the control arm, then a two-group randomizedcontrolled clinical trial of a new therapy would need only 800 patients to find a $40 \%$ change in the event rate (i.e., a realistic relative effect size of 20\%), assuming conventional values for alpha and beta (0.05 and 0.2, respectively). This type and size of trial is certainly within the reach of networks of investigators studying CS-AKI, 
and these new data should renew the field's enthusiasm for interventional studies of CS-AKI.

One limitation of the study by Karkouti et al. is their assessment of only adult patients who underwent cardiopulmonary bypass. We cannot determine whether the $30 \%$ threshold value is valid for off-pump surgery or for use in children. Nevertheless, a validation study in a suitable dataset would not be too difficult to conduct, and investigators with access to such datasets might consider such a project worthwhile. Another limitation of this study is the delay of "diagnosis" until ICU admission, and thus, any putative intervention based on this criterion would be effective only when applied postoperatively and not intraoperatively. This salvage $v s$ prevention problem is not easily solved, however, and should not detract from the value of the current work.

The knowledge of an association between a 30\% bump in serum creatinine on admission to ICU after on-pump heart surgery and a $50 \%$ chance of developing AKI in the next $48 \mathrm{hr}$ will undoubtedly assist researchers in the design and conduct of interventional trials in CS-AKI. These new data not only provide research value but may also benefit clinicians. For instance, this test does not represent an expensive novel intervention, and inexpensive therapeutic interventions have recently been shown to reduce the incidence of AKI once these high-risk patients are identified. ${ }^{9}$ In their study, Meersch et al. reduced the incidence of AKI by following an algorithm designed to optimize hemodynamic conditions and minimize further renal risk factors. It is telling that the investigators did not establish a longer-term clinical benefit; however, suggesting the link between AKI and long-term outcomes needs further clarification. In summary, Karkouti et al. should be congratulated on expertly demonstrating how to repurpose a pair of existing research datasets in order to provide real progress in the design of future clinical trials.

\section{L'insuffisance rénale aiguë associée à la chirurgie cardiaque: outils pour étoffer les populations d'études cliniques}

Le terme 'insuffisance rénale aiguë' (IRA) est devenu synonyme d'un déclin aigu de la fonction rénale, bien que les reins à proprement parler ne soient presque jamais « affectés de façon aiguë ». Lorsqu'une IRA survient peu après une chirurgie cardiaque, on parle alors d'IRA associée à la chirurgie cardiaque (IRA-CC) et, en raison de sa fréquence (jusqu'à $50 \%$ selon la définition utilisée et la population à l'étude), ${ }^{1,2}$ elle est devenue un critère d'évaluation très intéressant tant pour les cliniciens que les chercheurs. D'importants efforts ont été déployés afin de parvenir à une définition consensuelle - convergeant récemment vers la définition retenue dans l'étude KDIGO (pour Kidney Disease: Improving Global Outcomes, soit 'La maladie rénale : pour l'amélioration des pronostics globaux') ${ }^{3}$ et récemment passée en revue par Schetz- ${ }^{4}$ et la controverse existe quant à savoir si les critères de diurèse augmentent systématiquement la précision diagnostique de ce système de définition. ${ }^{5}$

Toutefois, malgré l'attention importante dont jouit l'IRA-CC, aucun progrès notoire n'a été observé dans sa prévention ou son traitement. Voilà une source de frustration constante. À une époque où il est de plus en plus difficile d'obtenir du financement pour la recherche, nous devons trouver des façons d'augmenter les chances que les études qui bénéficient de financement rencontrent le succès. L'une des manières d'y parvenir est de n'inclure que les patients dont la maladie est connue et établie dans les études prospectives d'intervention. De cette façon, nous omettons les personnes qui ne souffrent pas de la maladie et qui ne peuvent donc pas bénéficier de l'intervention à l'étude. Qui plus est, les coûts sont ainsi réduits et nous augmentons les chances de trouver un traitement efficace. Cependant, ce modus operandi écarte également la possibilité de la prévention, qui constitue, de manière générale, une 'intervention' plus désirable, particulièrement si nous parvenons à identifier un groupe de patients courant un risque accru d'être frappés par la maladie à l'étude. Dans l'idéal, nous aimerions pouvoir identifier, de façon prospective, une population de patients courant un risque élevé de manifester un pronostic pour ensuite n'inclure que ces patients dans une étude clinique. Zarbock et coll. ${ }^{6}$ ont montré le succès de cette approche dans les cas d'IRA-CC en combinant un enrichissement clinique et des mesures des biomarqueurs dans l'urine.

Dans ce numéro du Journal, Karkouti et coll. ${ }^{7}$ rapportent une étude observationnelle qui se fonde sur une augmentation postopératoire immédiate de la créatininémie pour déterminer à l'avance un sousensemble de patients qui manifestera plus tard une IRACC. La conception de l'étude est robuste et, plus important encore, les chercheurs se sont servis d'un ensemble de données complètement indépendant pour valider leurs travaux. Ainsi, les auteurs ont pu contourner la problématique habituelle liée à l'ajustement excessif du modèle, la généralisation externe, et la pertinence dans le «monde réel », particulièrement étant donné que l'ensemble de données de validation est dérivé d'une étude clinique multicentrique pragmatique plus ancienne. ${ }^{8}$ Dans l'étude présentée ici, les auteurs rapportent qu'une augmentation immédiate (c.-à-d., au moment de 
l'admission à l'unité de soins intensifs [USI]) de $30 \%$ de la créatininémie permettait d'identifier une population de patients courant un risque élevé de souffrir d'IRA au cours des prochaines $48 \mathrm{~h}$. La valeur prédictive positive dans l'ensemble de validation était de $55 \%$ (autrement dit, une personne avec une augmentation de $30 \%$ de la créatininémie lors de son admission à l'USI court environ une chance sur deux de souffrir d'une IRA au cours des prochaines $48 \mathrm{~h}$ ), et la valeur prédictive négative était de 92 $\%$ (donc, une personne sans augmentation de $30 \%$ ne souffrira pas d'IRA de façon quasi certaine). Cette information pourrait être très utile pour les chercheurs planifiant des études d'intervention en IRA-CC. Si nous pouvons, pour l'essentiel, garantir un taux d'événements de $50 \%$ dans le groupe témoin, alors une étude clinique randomisée contrôlée à deux groupes examinant un nouveau traitement ne nécessiterait que quelques 800 patients pour mettre en lumière un changement de $40 \%$ dans le taux d'événements (c.-à-d. une taille d'effet relative réaliste de $20 \%$ ), si l'on prend pour acquis des valeurs alpha et beta conventionnelles $(0,05$ et 0,2 , respectivement). Une étude de ce type et de cette envergure est indubitablement à la portée des réseaux de chercheurs étudiant l'IRA-CC, et ces nouvelles données devraient redonner un peu d'enthousiasme au milieu par rapport aux études d'intervention sur l'IRA-CC.

L'un des écueils de l'étude de Karkouti et coll. est qu'elle n'évalue que des patients adultes opérés sous circulation extracorporelle. Il est par conséquent impossible de déterminer si la valeur seuil de $30 \%$ s'appliquerait pour les chirurgies à cœur battant ou si elle peut être utilisée chez les enfants. Ceci étant dit, une étude de validation dans un ensemble de données approprié ne serait pas trop difficile à réaliser - et des chercheurs ayant accès à de tels ensembles de données pourraient voir la valeur d'un tel projet. Une autre limitation de cette étude est le délai du « diagnostic » jusqu'à l'admission à l'USI : en effet, toute intervention putative fondée sur ce critère ne serait efficace que lorsqu'elle serait appliquée en période postopératoire, et non peropératoire. Cette question du sauvetage $v s$ prévention n'est toutefois pas facile à résoudre et cela ne devrait rien enlever à la valeur de ces travaux.

La connaissance d'une association entre une augmentation de $30 \%$ de la créatininémie à l'admission à l'USI après une chirurgie cardiaque sous circulation extracorporelle et une chance de $50 \%$ de souffrir d'IRA au cours des prochaines $48 \mathrm{~h}$ aidera certainement les chercheurs lors de la conception et de la réalisation d'études d'intervention en IRA-CC. Ces nouvelles données ont non seulement une valeur du point de vue de la recherche, mais elles pourraient également être utiles aux cliniciens. Par exemple, ce test ne représente pas une intervention innovante onéreuse, et il a récemment été démontré que les interventions thérapeutiques peu onéreuses réduisaient l'incidence d'IRA une fois ces patients à risque élevé identifiés. ${ }^{9}$ Dans leur étude, Meersch et coll. ont réduit l'incidence d'IRA en suivant un algorithme conçu pour optimiser les conditions hémodynamiques et minimiser les facteurs de risque rénaux supplémentaires. Fait marquant, les chercheurs n'ont pas établi de bienfait clinique à plus long terme toutefois, ce qui laisse penser que le lien entre l'IRA et les pronostics à long terme devra faire l'objet d'un examen plus approfondi. En résumé, il convient de féliciter Karkouti et coll. car ils ont su démontrer, de façon experte, comment réutiliser deux ensembles de données de recherche existants afin d'accomplir des progrès réels dans la conception d'études cliniques futures.

Conflicts of interest None declared.

Editorial responsibility This submission was handled by Dr. Philip M. Jones, Associate Editor, Canadian Journal of Anesthesia.

Disclosure Dr. Shaw is a consultant for Astute Medical ${ }^{\circledR}$.

\section{Conflit d'intérêt Aucun.}

Responsabilité éditoriale Cet article a été traité par Dr Philip M. Jones, rédacteur adjoint, Journal canadien d'anesthésie.

Déclaration Dr Shaw est consultant pour Astute Medical ${ }^{\circledR}$.

\section{References}

1. Fuhrman DY, Kellum JA. Epidemiology and pathophysiology of cardiac surgery-associated acute kidney injury. Curr Opin Anaesthesiol 2017; 30: 60-5.

2. Hoste EA, Cruz DN, Davenport A, et al. The epidemiology of cardiac surgery-associated acute kidney injury. Int J Artif Organs 2008; 31: 158-65.

3. Kidney Disease: Improving Global Outcomes (KDIGO) Acute Kidney Injury Work Group. KDIGO clinical practice guidelines for acute kidney injury: Section 2: AKI definition. Kidney Int Suppl 2012; 2: 19-36.

4. Schetz M, Schortgen $F$. Ten shortcomings of the current definition of AKI. Intensive Care Med 2017. DOI:10.1007/s00134-017-4715-2.

5. Koeze J, Keus F, Dieperink W, van der Horst IC, Zijlstra JG, van Meurs $M$. Incidence, timing and outcome of AKI in critically ill patients varies with the definition used and the addition of urine output criteria. BMC Nephrol 2017; 18: 70.

6. Zarbock A, Schmidt C, Van Aken H, et al. Effect of remote ischemic preconditioning on kidney injury among high-risk patients undergoing cardiac surgery: a randomized clinical trial. JAMA 2015; 313: 2133-41.

7. Karkouti K, Rao V, Chan CT, Wijeysundera DN; TACS Investigators. Early rise in postoperative creatinine for identification of acute kidney injury after cardiac surgery. Can J Anesth 2017. DOI:10.1007/s12630-017-0899-8 
8. Karkouti K, Callum J, Wijeysundera DN, et al. Point-of-care hemostatic testing in cardiac surgery: a stepped-wedge clustered randomized controlled trial. Circulation 2016; 134: 1152-62.

9. Meersch M, Schmidt C, Hoffmeier A, et al. Prevention of cardiac surgery-associated AKI by implementing the KDIGO guidelines in high risk patients identified by biomarkers: the PrevAKI randomized controlled trial. Intensive Care Med 2017. DOI:10. 1007/s00134-016-4670-3. 